Italy; ${ }^{10}$ Univ, Strasbourg, France; ${ }^{11} \mathrm{CMC}$, Vellore, India; ${ }^{12}$ Sapienza Univ, Rome; ${ }^{13}$ Santa Maria, Udine, Italy; ${ }^{14}$ INCMNSZ, México City, Mexico; ${ }^{15}$ UMC, Utrecht, Netherlands; ${ }^{16}$ Catholic Univ Korea, Seoul, Korea, Republic Of; ${ }^{17}$ Karolinska Instit, Stockholm, Sweden; ${ }^{18}$ UMCL, Ljubliana, Slovenia; ${ }^{19}$ Lariboisière Hosp, Paris, France; ${ }^{20}$ Univ, Perugia, Italy; ${ }^{21}$ Hosp Vall d'Hebron, Barcelona, Spain; ${ }^{22}$ Univ Hosp, Kanazawa, Japan; ${ }^{23}$ UCL, London, United Kingdom; ${ }^{24}$ TQEH, Adelaide, Australia; ${ }^{25}$ Univ, Uppsala, Sweden; ${ }^{26}$ Hosp Ramón y Cajal, Madrid, Spain; ${ }^{27}$ Med Hosp, Wroclaw, Poland; ${ }^{28}$ Univ, Groningen, Netherlands; ${ }^{29}$ Univ, Nagasaki, Japan; ${ }^{30}$ UFES, Vitória, Brazil; ${ }^{11}$ Univ, L'Aquila, Italy; ${ }^{32}$ Univ, Brest, France; ${ }^{33}$ TUM, München, Germany; ${ }^{34}$ QMUL, London, United Kingdom; ${ }^{35}$ UNIFESP, Sao Paulo, Brazil; ${ }^{36}$ Haukeland Hosp, Bergen, Norway; ${ }^{37}$ School Med SBU, Mineola, United States; ${ }^{38}$ USP, Sao Paulo, Brazil; ${ }^{39}$ Univ, Montpellier, France; ${ }^{40}$ INICSA, Cordoba, Argentina; ${ }^{41}$ Univ, Cairo, Egypt; ${ }^{42}$ L.Sacco Univ, Milan, Italy; ${ }^{43}$ German Hosp, Buenos Aires, Argentina

Objectives: Baseline characterization of European patients diagnosed with primary Sjögren syndrome (SS) according to the $2002 \mathrm{AE}$ criteria.

Methods: The Big Data Sjögren Project was formed in 2014 to take a "highdefinition" picture of the main features of primary SS by merging international SS databases. International experts of the EULAR-SS Task Force were invited to participate. By January 2017, the database included 9302 consecutive patients recruited from 21 countries of the 5 continents.

Results: A total of $6586(71 \%)$ patients were included from European countries. In comparison with non-European countries, European patients had a higher mean age (54 v 51yrs, $p<0.001)$, higher frequency of men $(7 \% \vee 5 \%, p=0.001)$, dry eyes ( $94 \%$ vs $88 \%, p<0.001)$, dry mouth ( $94 \%$ vs $91 \%, p<0.001)$, and lower frequency of abnormal ocular ( 84 vs $86 \%, p=0.049$ ) and oral (75 vs $81 \%, p<0.001$ ) tests. Immunologically, European patients had a lower frequency of anti-Ro/La antibodies (69 vs $78 \%, p<0.001$ ) and a higher frequency of RF (50 vs $47 \%$, $p=0.01)$, low C4 (14 vs $9 \%, p<0.001)$ and cryoglobulins $(8 \%$ vs $3 \%, p<0.001)$. Logistic regression identified as independent variables older age (OR 1.02), male gender (OR 2.62), abnormal oral tests (OR 0.26), anti-Ro/La antibodies (OR 0.69), RF (OR 1.76), low C4 (OR 1.97) and cryoglobulins (OR 3.85).

Conclusions: European patients are diagnosed at older age, are more frequently men, and presented a lower frequency of anti-Ro/La antibodies and a higher frequency of immunological markers related to mixed cryoglobulinemia.

Disclosure of Interest: None declared

DOI: 10.1136/annrheumdis-2017-eular.3502

\section{SAT0303 CLINICAL DIFFERENCES BETWEEN DEFINITE AND PROBABLE ANTIPHOSPHOLIPID (APS) PATIENTS: SHOULD THEY BE TREATED THE SAME?}

F. Signorelli, G. Balbi, R.A. Levy. Rheumatology, Universidade do Estado do Rio de Janeiro, Rio de Janeiro, Brazil

Background: The management of patients with recurrent thromboses/pregnancy morbidity and transient detection of antiphospholipids antibodies (aPL) can be a medical challenge. Although these patients are commonly seen in practice, there are no specific guidelines for the treatment in this situations.

Objectives: To investigate the clinical differences between definite and probable APS patients.

Methods: We performed a cross-sectional study in a group of 90 outpatients seen in our department. Seventy-seven of them met the Sydney classficiation criteria for definte APS, and thirteen had thrombosis or gestational morbidity, but no definite serological criteria for the diagnosis of APS. Clinical and serological features were collected during visits and by chart review, and the two groups were compared. Transient aPL was defined as only one detection of aPL (lupus anticoagulant, anticardiolipin $\mathrm{lgM} / \mathrm{lgG}$ and/or anti-beta-2-glycoprotein $1 \mathrm{lgM} / \mathrm{lgG}$ ) after 2 or more assays, at least 12 weeks apart.

Results: Demographic and clinical characteristics are shown in Table 1. In a bivariate analysis, there was no difference between groups regarding the criteria and non-criteria manifestations of APS, except for the presence of livedo $(p=0.033)$. In a multivariate regression analysis, the model was adjusted to age, sex, and variables with $p<0.10$ in the bivariate analysis (age, sex, race, livedo,

Table 1. Demographic and clinical characteristics $(\mathrm{N}=90)$

\begin{tabular}{lccc}
\hline Variable & Definite APS $(\mathrm{N}=77)$ & Probable APS $(\mathrm{N}=13)$ & P value \\
\hline Age & $42.0 \pm 12$ & $40.5 \pm 10.3$ & $\mathrm{NS}$ \\
Female gender & $64(83.1)$ & $12(92.3)$ & $\mathrm{NS}$ \\
Caucasian & $50(64.9)$ & $8(61.5)$ & $\mathrm{NS}$ \\
Time first manifestation (mo) & $120(33.3-50)$ & $174(81-202)$ & $\mathrm{NS}$ \\
& Criteria manifestations & & \\
Thrombotic & $72(93.5)$ & $13(100)$ & $\mathrm{NS}$ \\
Obstetric & $28(43.8)^{\star}$ & $1(8.3)^{\star \star}$ & $\mathrm{NS}$ \\
& $\mathrm{Non}$ criteria & & \\
Livedo & $20(26)$ & $0(0)$ & $\mathrm{p}=0.033$ \\
Thrombocytopenia & $11(14.3)$ & $0(0)$ & $\mathrm{NS}$ \\
Valvulopathy & $7(11.7)^{\ddagger}$ & $1(11.1)^{\ddagger \ddagger}$ & $\mathrm{NS}$ \\
Raynaud phenomenon & $21(27.3)$ & $1(7.7)$ & $\mathrm{NS}$ \\
Migraine & $36(46.8)$ & $8(61.5)$ & $\mathrm{NS}$ \\
\hline
\end{tabular}

${ }^{*} \mathrm{~N}=64 ;{ }^{* \star} \mathrm{N}=12,{ }^{\ddagger} \mathrm{N}=60,{ }^{\ddagger} \mathrm{N}=9 . \mathrm{Mo}=$ months. $\mathrm{CVD}=$ cardiovascular disease. Values showed as $\mathrm{N}(\%)$ for categorical variables, mean $\pm \mathrm{SD}$ for normal distribution and Median (interquartil range) for asymmetric distribution.
Raynaud's phenomenon). No difference between groups was found after the analysis.

Conclusions: This study suggests patients with transient detection of aPL have the same clinical characteristics of patients with definite APS, including thrombotic features, pregnancy morbidity, and non-criteria manifestations. In this context, our data suggests that both groups should be treated according to the current treatment guidelines for APS.

Disclosure of Interest: None declared

DOI: 10.1136/annrheumdis-2017-eular.6987

\section{SAT0304 STIMULATORY AND INHIBITORY KILLER IMMUNOGLOBULIN-LIKE RECEPTORS ON NATURAL KILLER $T$ (NKT) CELLS IN PATIENTS WITH SYSTEMIC LUPUS ERYTHEMATOSUS (SLE): RELATION TO DISEASE ACTIVITY}

S.I. Nasef ${ }^{1}$, A.M. Asker ${ }^{2}$, H.H. Omar ${ }^{2}$, H.M. Hassoba ${ }^{2} .{ }^{1}$ Physical Medicine, Rheumatology and Rehabilitation; ${ }^{2}$ Clinical Pathology, Faculty of Medicine, Suez Canal University, Ismailia, Egypt

Background: Natural killer T (NKT) cells are a unique subgroup of $\mathrm{T}$ cells that represents a bridge between innate and adaptive immunity. The functions of NKT-cells are regulated by the balance between activating and inhibitory Killer cell immunoglobulin-like receptors (KIRs). Systemic lupus erythematosus (SLE) patients showed aberrant expression of KIRs on NKT-cells. Whether the expression pattern of KIRs on NKT-cells is associated with disease activity in SLE is still unknown.

Objectives: Assessment of expression of stimulatory and inhibitory KIRs on NKT-cells in SLE patients and its relation to disease activity.

Methods: We recruited 40 SLE patients and 20 age and gender matched healthy controls. According to SLE disease activity index (SLEDAI), patients were divided into two groups; active SLE $(n=20)$ and inactive SLE $(n=20)$. SLE was active when SLEDAI was $\geq 4$. Immuno-phenotyping by flow cytometry was done using markers for NKT-cells (CD3 and CD56), stimulatory KIRs (KIR2DL4, CD158D) and inhibitory KIRs (KIR3DL1, CD158E1). Absolute counts and percentage of NKT cells expressing CD158D and CD158E1 together with their mean fluorescence intensity (MFI) were measured. The histogram of CD158 expression was used to assess KIRs on NKT cells.

Flow cytometry charts of lymphocytes and NKT cells in SLE patients (active and inactive) and controls are shown in (Figure 1).

Laboratory work included ANA, anti-dsDNA, Anti-smith, C3, C4, CRP and ESR.

Results: Mean age of patients was $29.9 \pm 10.8$ years. Females constituted $95 \%$ $(n=38)$ of patients. Mean disease duration was $4.4 \pm 4.5$ years. Mean SLEDAI was $11.75 \pm 7.43$.

NKT-cells absolute number (cell/ $/ \mathrm{mm}^{3}$ ) was significantly decreased in SLE patients $(89 \pm 135)$ compared to controls $(287 \pm 133),(P=0.001)$. Likewise, the absolute number of NKT-cells $\left(\right.$ cell $\left./ \mathrm{mm}^{3}\right)$ was significantly lower in active SLE patients $(65.2 \pm 57.8)$ compared to inactive ones (114.5 \pm 181.1$),(P=0.001)$.
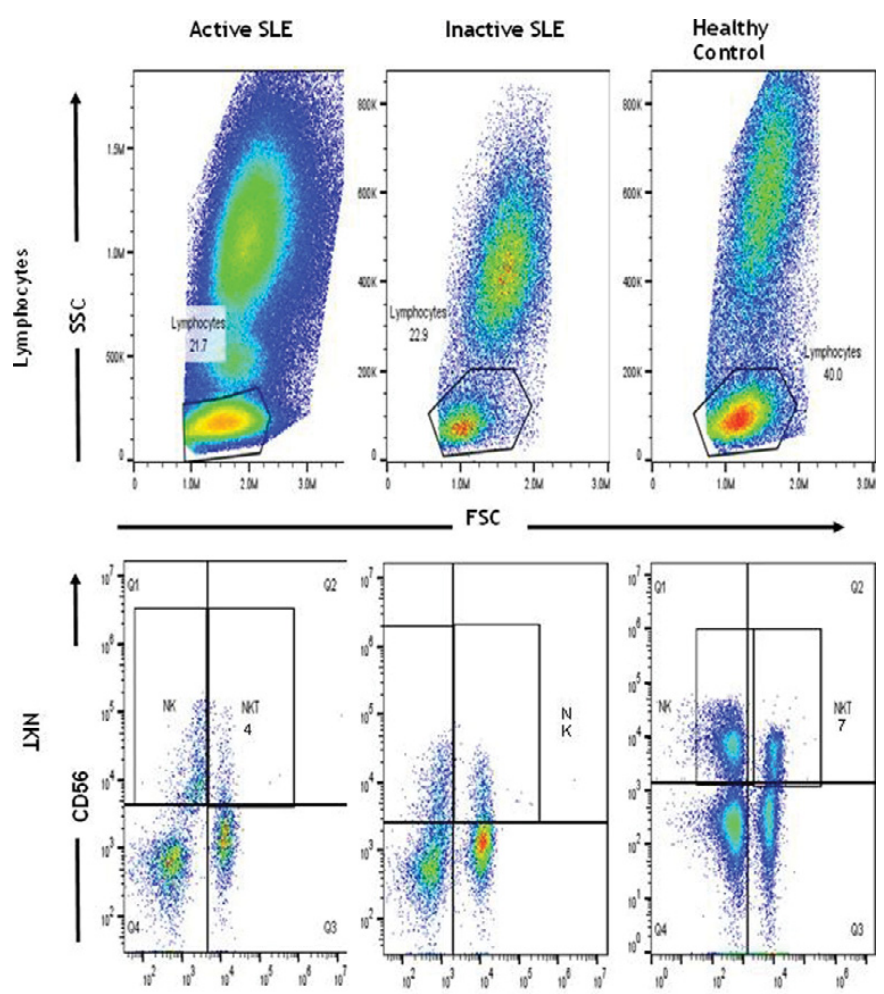

FSC
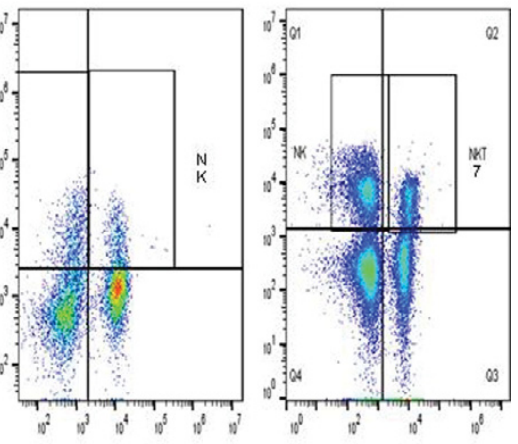

CD3 
The expression of inhibitory KIRs was significantly lower in SLE patients $(2.83 \pm 2.8 \%)$ compared to controls $(5.54 \pm 2.01 \%) \quad(P=0.001)$ While, stimulatory KIRs were significantly higher in SLE patients $(3.2 \pm 3.7 \%)$ than controls $(1.02 \pm 0.5 \%),(P=0.001)$. Active SLE patients showed significantly increased expression of stimulatory KIRs $(5.29 \pm 4.29 \%)$ than inactive patients $(1.13 \pm 0.89 \%)$, $(P=0.004)$. However, inhibitory KIRs were significantly decreased in active $(1.28 \pm 1.32 \%)$ than inactive SLE patients $(4.38 \pm 3.05 \%),(p=0.003)$.

Expression of stimulatory KIRs correlated positively with ESR $(r=0.3, P=0.04)$ and negatively with $\mathrm{C} 4(r=-0.4, P=0.01)$. In contrast, inhibitory KIRs correlated negatively with $\mathrm{ESR}(r=-0.5, P=0.003)$ and positively with $\mathrm{C} 4(r=0.4, P=0.02)$. Using Receiver operating characteristic (ROC) curve analysis, expression of inhibitory KIRs on NKT-cells predicted disease activity at a cut-off value of $\leq 1.7 \%$ with $80 \%$ sensitivity and $80 \%$ specificity $(P=0.001)$. While, expression of stimulatory KIRs on NKT-cells predicted active disease at a cut-off value of $>1.4 \%$, sensitivity $(85 \%)$, and specificity $(80 \%)(P=0.001)$.

Conclusions: SLE activity is associated with an increased expression of stimulatory KIRs as well as a decreased expression of inhibitory KIRs on NKT cells. This may play a role in the pathogenesis of flares and acceleration of disease activity in SLE and could be a therapeutic target for SLE patients. Disclosure of Interest: None declared

DOI: 10.1136/annrheumdis-2017-eular.3920

\section{SAT0305 HISTOLOGY OF MINOR SALIVARY GLANDS IN PATIENTS WITH SJÖGREN'S SYNDROME, ASSOCIATION WITH CLINICAL AND LABORATORY ASPECTS}

S. Colafrancesco ${ }^{1}$, F. Arienzo ${ }^{1}$, B. Cerbelli ${ }^{2}$, A. Gattamelata ${ }^{1}$, A. Minniti ${ }^{1}$, G. Picarelli ${ }^{1}$, C. Giordano ${ }^{2}$, G. D'Amati ${ }^{2}$, R. Priori ${ }^{1}$, G. Valesini ${ }^{1} .{ }^{1}$ Dipartimento di medicina interna e specialità mediche; ${ }^{2}$ Dipartimento di radiologia, Oncologia e Scienze Radiologiche, Sapienza University of Rome, Rome, Italy

Background: Minor salivary gland (MSG) biopsy represents an useful tool not only for the diagnosis of primary Sjogren's Syndrome (pSS) but also to evaluate patients prognosis. Recognition of germinal centers (GCs) by hematoxilin eosin (HE) and/or $\mathrm{IHC}$ staining for follicular dendritic cells (FDC) detection is mandatory, representing a risk factor for lymphoma development. Focus score (FS) is one of the main instrument to quantify MSG impairment, nonetheless quality information regarding the type of infiltrate such as the entity, structure and localization, are lacking.

Objectives: Aim of this study is to find any association of specific histological features of MSG from patients with pSS with the principal clinical and laboratory features. Moreover, to investigate the utility of histological parameters, other than FS or GCs, for characterizing patients.

Methods: Patients with pSS were enrolled in our SS clinic, and clinical/laboratory data (table) referring to the time of MSG biopsy, gathered on a dedicated database. MSG, removed for diagnostic purposes, were preserved as paraffin embedded tissue, then cut and sequentially stained by $\mathrm{H} \& \mathrm{E}$ and IHC [polyclonal rabbit anti-CD3 (lymphocytes T); monoclonal mouse anti-CD20 (lymphocytes B); monoclonal mouse anti-CD21 (FDC)]. Images were collected by Zeiss Axio Scan and analysed (ZEN software) as follows: FS calculation, mean foci area, percentage of infiltration, presence of segregated foci (SF) (specifically, clear evidence of $T$ and $B$ cells area by CD3-CD20 double staining), GCs and lymphoepithelial lesions (LELs) detection.

Results: 53 MSG from patients with pSS were collected and analysed. Patients clinical and laboratory data are reported in table. FS positively correlated with the percentage of infiltration $(p<0.001)$ as well as with the presence of SF $(p=0.005)$, GCs $(p=0.02)$ and LELs $(P=0.005)$. Mean foci area and percentage of infiltration correlated with SF $(p=0.0002$ and $p<0.001$, respectively), GCs $(p=0.0004$ and $p<0.001$, respectively) and LELs (both $p<0.001$ ). SF correlated with GCs and

\begin{tabular}{ll}
\hline Clinical features & Number $\%$ ) \\
Sex (M/F) & $4 / 49$ \\
Mean age at diagnosis (mean士SD, years) & $54.9 \pm 12.1$ \\
Xerophtalmia & $47 / 53(88.6)$ \\
Xerostomia & $47 / 53(88.6)$ \\
Salivary gland swelling & $16 / 53(30.1)$ \\
Arthritis & $8 / 53(15)$ \\
Lymphoma & $0 / 53(0)$ \\
Purpura & $2 / 53(3.7)$ \\
PNS and/or CNS involvement & $1 / 53(1.8)$ \\
Pancreatitis & $1 / 53(1.8)$ \\
Laboratory features & \\
ANA & $39 / 53(73.6)$ \\
Anti-Ro/SSA & $17 / 53(32.1)$ \\
Anti-La/SSB & $14 / 53(26.4)$ \\
Hypergammaglobulinaemia & $18 / 53(33.9)$ \\
Rheumatoidfactor & $10 / 53(19)$ \\
Leukopenia & $8 / 53(15)$ \\
Hypocomplementemia & $6 / 53(11.3)$ \\
Monoclonal component & $8 / 53(15)$ \\
Cryoglobulinaemia & $2 / 53(3.7)$ \\
\hline
\end{tabular}

LEL $(\mathrm{p}<0.001)$. Anti nuclear antibodies $(A N A)$ were associated with the presence of $S F(p=0.029, O R=5.7 \mathrm{Cl}=1.1-28.8)$ while gland swelling was associated with the presence of $\mathrm{GCs}(\mathrm{p}=0.043$, OR=4, $\mathrm{Cl}=1.1-15)$.

Conclusions: The FS was associated with the presence of GCs and LELs, as well as with more organized infiltrates characterized by segregation in $T$ and $\mathrm{B}$ areas (SF), thus representing an useful tool which mirrors the risk of lymphoma. From our study, the qualitative characteristics of the biopsy, including SF, percentage of infiltration or the mean foci area, appear to be strictly linked. Moreover, their association with the presence of GCs and LELs supports the importance to consider also these features during histological examination. The lack of correlation between histological parameters and clinical/laboratory features might reveal a weaker connection between histological findings and specific SS phenotypes except for the relationship between glandular swelling and GCs which confirms how this clinical aspect should be considered as a risk factor for lymphoma development.

Disclosure of Interest: None declared

DOI: 10.1136/annrheumdis-2017-eular.5862

\section{SAT0306 ASSOCIATION BETWEEN QUALITY OF SLEEP, QUALITY OF LIFE AND DISEASE ACTIVITY IN PATIENTS WITH SYSTEMIC LUPUS ERYTHEMATOSUS}

S. Monov $^{1}$, D. Monova ${ }^{2}$, M. Ivanova ${ }^{3} .{ }^{1}$ Department of Rheumatology, Medical University - Sofia; ${ }^{2}$ Department of Internal Diseases, Medical University - Sofia, Medical Institute; ${ }^{3}$ Department of Internal Diseases, Medical Institute, Sofia, Bulgaria

Background: Systemic Lupus Erythematosus (SLE) patients are known to have sleep disturbances. Quality of sleep may affect quality of life, but this association has not been systematically evaluated.

Objectives: The aim of this study was to examine the association of quality of sleep, quality of life and SLE disease activity in patients diagnosed with SLE.

Methods: 132 SLE patients with a confirmed diagnosis of SLE according to the ACR classification criteria were enrolled in this study. The patients completed the following questionnaires: the Pittsburgh Sleep Quality Index (PSQI), the 12 item Short Form Health Survey (SF-12), the Lupus Patient-Reported Outcome tool (LupusPRO), SLE Quality of Life Questionnaire (SLE - QoL). Clinical information, including the SLE Disease Activity Index (SLEDAI), was obtained from medical records. Student's t-test, ANOVA, Pearson correlation measured were used in statistical analysis.

Results: The majority of the participants $(84,4 \%)$ had sleep disturbances (PSQI $>5$ ). Total PSQI score was weakly associated with all of the SF-12 subcategories and showed weak to moderate associations with the LupusPRO subcategories $(r<0,05)$, except for "medication" $(r<0,20)$. "Sleep duration" was not associated with any of the SF-12 or LupusPRO subcategories. "Sleep efficiency" was weakly associated with "physical health", "physical function", and "pain" in the SF-12 and LupusPRO. "Sleep quality" and "sleep disturbances" were weakly associated with "pain" and the "emotional" and "mental" subcategories in the SF-12 and LupusPRO. SLE - QoL was significantly higher in patients with good sleep.

Conclusions: We found that quality of sleep, especially "sleep efficiency", was poor for the majority of patients with SLE. Quality of sleep was associated with various aspects of quality of life, especially pain, vitality, and emotional health. Management of pain and emotional health may be important for improving quality of sleep in SLE patients.

Disclosure of Interest: None declared

DOI: 10.1136/annrheumdis-2017-eular.1450

\section{SAT0307 RECURRENCE RATE OF THROMBOSIS FOR PATIENTS WITH ANTI-PHOSPHOLIPID ANTIBODIES INITIALLY AND DISAPPEARED LATER AFTER THROMBOSIS}

S.M. Kim ${ }^{1}$, J. Kim ${ }^{2}$, Y. Kim ${ }^{3}$, S.W. Kang ${ }^{2}$, S.-C. Shim ${ }^{2}$, S.-J. Yoo ${ }^{3} .{ }^{1}$ Internal Medicine, Chungbuk National University Hospital, Cheong-ju; ${ }^{2}$ Internal Medicine, School of Medicine Chungnam National University; ${ }^{3}$ Internal Medicine, Chungnam National University Hospital, Daejeon, Korea, Republic Of

Background: In case of anti-phospholipid syndrome, anticoagulants are recommended. However, there were no data about recurrence rate for thrombosis in patients with anti-phospholipid antibodies (APS) initially which disappeared later. Objectives: We compared recurrence rate of thrombosis between negative conversion group and control group.

Methods: We reviewed the medical records of patients diagnosed with thrombosis such as cerebral infarct, myocardial infarct, deep vein thrombosis, or thrombosis of other vessels at a tertiary medical center from 2000 to October 2016. Of these, 14 patients whose APA status was converted from positive to negative after more than 12 weeks were enrolled as negative conversion group. Forty-six patients without APA were matched with the ratio of 1:3 1:4 according to age, sex, thrombosis type (arterial or venous) and therapeutic agents as control group. Results: There was no difference between negative conversion group and control group in smoking status, presence of diabetes or hypertension, duration from the thrombosis to last visits or to recurrence, the proportion of patients taking glucocorticoids. There was no difference in the overall recurrence of thrombosis between two groups [negative conversion group, $3 / 14$ (21\%) vs. control group, 